\title{
IDS40: Reflections from Tanzania
}

\section{Roy Trivedy}

\author{
1 Introduction \\ I returned to IDS for the fortieth anniversary \\ conference after an absence of almost 20 years. \\ Feeling a sense of excitement tinged with \\ anticipation, I looked forward to hearing about \\ development challenges identified through the 40 \\ global Roundtable meetings, contributing to \\ discussions about whether development research \\ needed reinventing and how IDS could help to achieve \\ this.
}

The two-and-a-half-day event was enjoyable, challenging and stimulating. It provided the opportunity to learn more about global development challenges, share experience with colleagues from diverse backgrounds and cultures, renew old friendships and make new contacts.

\section{Common themes}

Many of the themes discussed in the Tanzania Roundtable were amplified in the presentations and discussions at the conference. It was reassuring to hear that many of the themes we had identified in Tanzania were also regarded as priorities for development research in other contexts. These included: the differential impact of globalisation; increasing global differentiation and inequalities (including diversity between developing countries); the lack of viable development choices and the reduced room for manoeuvre for many marginalised groups and states; diversity of development pathways; and the need to strengthen accountability of global institutions.

In addition, recurrent themes consistent with those identified in the Tanzania Roundtable specifically about the role of development research institutes included:

- the need to make development research more relevant to peoples' lives
- the need for research institutes in the North to be more aware of how research agendas are set, funded and carried out, and the need to build the capacity of 'Southern partners' to advocate for effective policy choices

- the need to ensure that research findings are communicated effectively and used to inform citizens so that they are better able to exert pressure on their governments, institutions and policymakers

- the need to build stronger linkages between policymakers, researchers and practitioners.

Many of these themes were elaborated upon by the speakers, and it was particularly interesting to hear Lawrence Haddad outline how these would be reflected in the future work programme of the IDS. I was particularly struck by the broad consensus on the need to make development research more relevant to local and global development challenges. Key points that I noted were the need for IDS and its sister institutions to:

- Provide citizens with evidence that enables them to hold policymakers, governments and institutions to account

- Contribute more effectively to social transformation, improve people's lives and expand choices available to the poor and marginalised

- Increase capacities in developing countries and enable the poor to carry out research based on their priorities.

\section{Some omissions}

I was surprised to hear relatively little discussion on some themes which take up a fair amount of my own 
time in the DFID office in Tanzania, and I suspect that of others working in official aid agencies.

This is a long list - which reflects the challenges facing those of us working at a practical level. Issues such as: the pros and cons of moving towards greater country ownership and country-led development; alternatives to state-led development; how to enable the private sector to play a greater role in promoting broad-based growth and development; how to improve multilateral and bilateral aid effectiveness; the pros and cons of changing aid modalities, especially the move to budget support; the need to find effective ways of supporting development in fragile states; how to build stronger alliances and partnerships for change; how to support the next generation of global development practitioners and leaders; and the need to continually improve institutional accountability to stakeholders and demonstrate results.

I was left wondering whether these issues did not come up because other participants did not see them as priorities for development research or were perhaps just less concerned about them.

\section{Areas for further reflection}

For me, it was refreshing to hear about themes which did not emerge so strongly in our discussions in Tanzania. For example: the impact of climate chaos on future development; the way in which gender, sexuality and funding patterns are impacting on human development; the need for more comparative research (including North-South and South-South) and making connections between local and global issues; and the way in which the focus on governance is likely to change over the next decade. I was enthused by Mike Edwards' vision of transformational social and political research, more independent of funding fashions and the confines of the aid industry, which would be people-centred, based on academic rigour, and strongly grounded in public policy and action.

This made me think more critically about the way in which many development agencies currently support development research and the kinds of knowledge and experience it generates. I wondered how IDS and its sister organisations could respond to this challenge and how official aid organisations, such as DFID, could support such a transition.

\section{Putting the vision into practice}

The conference enabled me to critically reflect on my own work as well as that of IDS. I had arrived feeling a little tired and harbouring some doubts about whether individuals and institutions could make a genuine difference to development outcomes and global tends. I left with renewed energy, hope and enthusiasm - determined to make a bigger contribution.

Like other participants, I will be watching developments at IDS to see how the anniversary discussions are followed up. It was a fantastic event, which deserves strong practical action by all interested in making development research even more relevant and effective in the future. 